\title{
Palatal wound healing using a xenogeneic collagen matrix - histological outcomes of a randomized controlled clinical trial
}

Thoma, Daniel S ; Hilbe, Monika ; Bienz, Stefan P ; Sancho-Puchades, Manuel ; Hämmerle, Christoph H F ; Jung, Ronald E

Abstract: AIM The aim of this study was to test whether or not a collagen matrix can improve early wound healing compared to spontaneous healing based on histological and immunohistologic analyses. METHODS In 20 volunteers, $6 \mathrm{~mm}$ punch biopsies were harvested at the palate. A xenogeneic collagen matrix (XCM) was sutured in one site; the other one was left untreated (control). Biopsies with a diameter of $8 \mathrm{~mm}$ were subsequently obtained at 4, 8, 15 and 29 days and histological and immunohistologic analyses were performed. RESULTS At day 4 , wound bed keratinization amounted to $12.4 \pm 7.5 \%$ (control) and $18.0 \pm 10.2 \%$ (XCM). This increased up to day 8 (19.7 $\pm 25.5 \%$ control; $29.1 \pm 8.0 \% \mathrm{XCM})$ and reached complete keratinization at day 15 in both groups. The quantitative analyses of the superficial compartment measured an increase in the amount of granulation tissue (32-88\% control; $14-41 \% \mathrm{XCM}$ ) from day 4 to day 8 . Angiogenesis was first detected at 8 days. At day 29 , the amount of connective tissue in all compartments reached values similar to the native tissue at baseline. CONCLUSIONS The application of a XCM as a wound dressing on palatal wounds might be beneficial in the early stages of wound healing. Further research with a larger sample size is needed to confirm these results.

DOI: https://doi.org/10.1111/jcpe.12624

Posted at the Zurich Open Repository and Archive, University of Zurich

ZORA URL: https://doi.org/10.5167/uzh-127136

Journal Article

Accepted Version

Originally published at:

Thoma, Daniel S; Hilbe, Monika; Bienz, Stefan P; Sancho-Puchades, Manuel; Hämmerle, Christoph H F; Jung, Ronald E (2016). Palatal wound healing using a xenogeneic collagen matrix - histological outcomes of a randomized controlled clinical trial. Journal of Clinical Periodontology, 43(12):1124-1131.

DOI: https://doi.org/10.1111/jcpe.12624 


\section{Palatal wound healing using a xenogeneic collagen matrix - histologic outcomes of a randomized controlled clinical trial}

Daniel S. Thoma ${ }^{a}$, Monika Hilbe ${ }^{b}$, Stefan Bienz, ${ }^{a}$ Manuel Sancho ${ }^{a}$, Christoph H. F. Hämmerle ${ }^{a}$, Ronald E. Jung ${ }^{a}$

Key words: $\quad$ wound healing, transplant (MeSH terms)

collagen matrix, soft tissue augmentation, keratinized tissue, substitute

Running title: Wound healing of a collagen matrix

${ }^{a}$ Clinic of Fixed and Removable Prosthodontics and Dental Material Science, Center of Dental Medicine, University of Zurich, Switzerland

${ }^{b}$ Institute of Veterinary Pathology, Winterthurerstrasse 268, 8057 Zuerich

Address for correspondence: PD Dr. Daniel S. Thoma

Clinic of Fixed and Removable Prosthodontics and Dental Material Science

Center of Dental Medicine, University of Zurich

Plattenstrasse 11, $\mathrm{CH}-8032$ Zurich, Switzerland

Phone: +41446343251

Fax: +41446344305

e-mail: daniel.thoma@zzm.uzh.ch 


\section{CONFLICT OF INTEREST AND SOURCE OF FUNDING STATEMENT}

This study was funded by the Clinic of Fixed and Removable Prosthodontics and Dental Material Science, University of Zurich, Switzerland and by a research grant from Geistlich Pharma AG, Wolhusen, Switzerland. The authors do not report any conflict of interest for this study. 


\section{Clinical Relevance}

Scientific rationale for the study: Collagen matrices (CM) were developed to increase the width of keratinized tissue around teeth and implants. This study presents the histologic analysis of the early healing of a xenogeneic collagen matrix (XCM) compared to a spontaneously healing experimental wound.

Principal findings: At 4 and 8 days, the extent of the epithelial coverage was slightly greater for XCM sites than for control sites. Furthermore, at 4 days and at 8 days after surgery there was a lower amount of granulation tissue found at XCM sites. At 15 days, all experimental wounds presented a fully keratinized epithelium.

Practical implications: The use of a xenogeneic collagen matrix as a palatal wound dressing may offer an accelerated wound healing at the early stages. 


\section{Abstract}

Aim: To test whether or not a collagen matrix can improve early wound healing compared to spontaneous healing based on histologic and immunohistologic analyses.

Methods: In 20 volunteers, $6 \mathrm{~mm}$ punch biopsies were harvested at the palate. A xenogeneic collagen matrix (XCM) was sutured in one site; the other one was left untreated (control). Biopsies with a diameter of $8 \mathrm{~mm}$ were subsequently obtained at 4 , 8,15 and 29 days and histologic and immunohistologic analyses were performed.

Results: At day 4, wound bed keratinization amounted to $12.4 \% \pm 7.5 \%$ (control) and $18.0 \% \pm 10.2 \%(X C M)$. This increased up to day $8(19.7 \% \pm 25.5 \%$ control; $29.1 \% \pm 8.0 \%$ XCM) and reached complete keratinization at day 15 in both groups. The quantitative analyses of the superficial compartment measured an increase of the amount of granulation tissue ( $32 \%$ to $88 \%$ control; $14 \%$ to $41 \% \mathrm{XCM}$ ) from day 4 to day 8 . Angiogenesis was first detected at 8 days. At day 29, the amount of connective tissue in all compartments reached values similar to the native tissue at baseline.

Conclusions: The application of a xenogeneic collagen matrix (XCM) as a wound dressing on palatal wounds might be beneficial in the early stages of wound healing. Further research with a larger sample size is needed to confirm these results. 


\section{INTRODUCTION}

Soft tissue grafting procedures are routinely performed for a variety of clinical indications in implant and reconstructive dentistry (Kim and Neiva, 2015). According to recent systematic reviews, the use of autogenous tissue harvested from the retromolar or palatal area is considered to be the gold standard around teeth and dental implants in partially edentulous patients (Thoma et al., 2014, Thoma et al., 2009, Levine et al., 2014, Cairo et al., 2014). Whereas these transplants and techniques are well documented, disadvantages are often reported, mostly due to the morbidity associated with the harvesting procedure and the respective second surgical site (Griffin et al., 2006, Wessel and Tatakis, 2008, Harris et al., 2005). In order to overcome these drawbacks, a number of soft tissue substitutes, predominantly derived from other medical fields, were introduced in dentistry (Liu et al., 2010). Namely collagen matrices made of collagen type I and III have been the field of interest in recent years. These substitute materials were evaluated in preclinical and clinical studies for gain of keratinized tissue around teeth and implants, for recession coverage, for ridge preservation and even for soft tissue volume gain (Schmitt et al., 2015, Rotundo and Pini-Prato, 2012, Nevins et al., 2011, Lorenzo et al., 2012, Jung et al., 2013b, Thoma et al., 2010, Cardaropoli et al., 2012). The currently available evidence suggests that these collagen matrices can be safely used, integrate well into surrounding tissue, increase tissue thickness and increase the width of keratinized tissue to an extent close to the gold standard, the autogenous tissue (Sanz et al., 2009, Thoma et al., 2012, Jung et al., 2011, Simion et al., 2012). The most often-used collagen matrix is designed for open healing. It consists of a loose matrix body specifically designed of reconstituted collagen fibers that should help to stabilize the blood clot and enhance connective tissue formation, whereas the superficial layer that is constituted of native collagen fibers is denser and is intended to enhance the migration of epithelial cells migrating from the border of the augmented site. This design is theoretically ideal and supported by preclinical and clinical data demonstrating the establishment of keratinized tissue in sites 
where collagen matrices are placed for open healing (Jung et al., 2011, Thoma et al., 2012, Schmitt et al., 2013). However, human histologic data are missing in terms of i) the early phases of healing using collagen matrices and, ii) possible advantages of using collagen matrices compared to spontaneous healing.

The aim of the present study was therefore to test whether or not a xenogeneic collagen matrix can improve early wound healing compared to spontaneous healing based on histologic and immunohistologic analyses. 


\section{Materials AND Methods}

\section{Study design}

The present study was performed as a randomized, controlled clinical trial using a splitmouth design. Following approval by the local ethics committee (Canton of Zurich, Zurich, Switzerland, KEK-ZH-Nr. 2011-0048), 20 volunteers (V1-20) were recruited and informed consent was obtained from all volunteers prior to the investigation. Subsequently, all volunteers were randomly allocated to one of four groups (V1-5 = primary endpoint at 4 days; $\mathrm{V} 6-10=$ at 8 days; $\mathrm{V} 11-15=$ at 15 days; $\mathrm{V} 16-20=$ at 29 days) according to a computer-generated randomization list. The study procedures were described in detail in a previous publication (Thoma et al., 2012).

\section{Surgical procedures}

On each side of the palate, two punch biopsies (diameter $6 \mathrm{~mm}$; depth $3 \mathrm{~mm}$ ) were harvested in all volunteers (V1-20) using a digitally designed stent (SMOP by Swissmeda, Swissmeda AG, Zurich, Switzerland) and a biopsy punch (Stiefel ${ }^{\circledR}$ Biopsy Punch, Stiefel Laboratorium $\mathrm{GmbH}$, Offenbach am Main, Germany), leaving the periosteum and part of the submucosa exposed (Figure $1 \mathrm{a}$ ).

The following two treatment modalities were then randomly applied to the defect sites according to a computer-generated randomization list:

- Wound closure with a xenogeneic collagen matrix (XCM; Geistlich Mucograft ${ }^{\circledR}$, Geistlich Pharma AG, Wolhusen, Switzerland) with a diameter of $6 \mathrm{~mm}$ and a thickness of $3 \mathrm{~mm}$. The XCM was sutured with non-resorbable sutures (Dafilon 60, Braun, Melsingen, Germany) (Figure $1 \mathrm{~b}$ ).

- Spontaneous healing, no further treatment (control). The defect was left to heal by secondary intention. 
The postoperative regimen included medication with an analgesics (Mefenamic acid, 500 mg every 8 hours) and a disinfectant solution (Chlorhexidine digluconate, $0.2 \%$ solution for oral rinsing every 8 hours for a period of 7 days). An individualized soft protective splint that completely covered the hard palate was given to all volunteers. Volunteers were instructed to use the splint during the first night to prevent bleeding and subsequently on an individual basis in case they felt pain. Sutures were removed at 8 days.

Follow-up

At $4,8,15$ and 29 days post surgery, all participants were recalled. According to the randomization schedule with 5 volunteers at each time-point, a second biopsy (diameter $8 \mathrm{~mm}$; depth $5 \mathrm{~mm}$ ) was harvested using an additional digitally designed stent. For volunteers 6-20, sutures were removed at 8 days.

All harvested biopsies were subjected to further histologic processing.

\section{Histologic processing}

After fixation, each sample was dehydrated in alcohol solutions of increasing concentration and embedded in paraffin. Embedded samples were cut at 2-3 $\mu \mathrm{m}$ thick slides using a microtome. Four sections per block were prepared: one slide was stained with van Gieson-elastica (VG-EL) for overall histological evaluation, three positive charged sections were used for immunohistochemical analyses.

\section{Histologic and histomorphometric analyses}

All histological sections (VG-EI) were evaluated using a Leica microscope (Leica DM6000 B, Wetzlar, Germany) for qualitative histological analysis. For histomorphometrical analysis, histological images were analyzed with a software program (Adobe Photoshop CS6). The selected region of interest (ROI) was divided into a superficial, central and apical compartment (Figure 2). 
Immunohistochemical analysis

Immunohistochemical analysis was performed to detect tissue remodeling and angiogenesis. Antibodies against mouse anti Vimentin (Clone Vim3B4; DAKO M3503, Switzerland), mouse anti Cytokeratin (Clone MNF116; DAKO M082101, Switzerland) and rabbit anti Factor VIII (DAKO A0082, Switzerland) (Figure 3) were used for antigen detection. As secondary antibody the DAKO REAL kit (K4005 HRP, Mouse AEC+ and K4009 HRP, Rabbit AEC+, DAKO, Switzerland) was used. Red staining (AEC 3-amino-9ethylcarbazole) indicated the presence of the protein. Positive and negative control sections were implemented. The negative control was achieved staining the slide without treatment of the primary antibody in order to evaluate false positive staining. Vimentin was used to detect mesenchymal cells like fibroblasts that form new collagen, cytokeratin to detect epithelial cells of the mucosa and factor VIII (von Willebrandt factor) to detect endothelial cells and therefore angiogenesis.

\section{Thickness of keratin layer and degree of epithelialization}

The thickness of the keratin layer in the defect area was categorized according to the following scale: regular $=>5$ layers, medium $=3-5$ layers, thin $(<3$ layers $)$ or absence of epithelium. Subsequently, linear measurements were performed calculating the surface each category occupied. Keratinization was considered if at least 3 layers of were present (medium and regular). One masked experienced examiner performed all the measurements.

\section{Quantitative analyses of regenerated tissue}

The following parameters were assessed as area percentages in a defined region of interest: i) epithelium (EP); ii) loose connective tissue (LCT); iii) dense connective tissue (DCT); iv) new formed granulation tissue (GT); v) fatty tissue (FT) vi) remaining collagen matrix material ( $\mathrm{rXCM})$; vii) background, any other remaining tissue within the region of interest. 


\section{Statistical analysis}

Descriptive statistics (means and standard deviations) were computed for the control and XCM sites, as well as for the difference between control and XCM values, at each time point and for each variable studied. No specific statistical tests were made due to a relatively low sample size and an increased number of volunteers compared to the previously published study including clinical patient-reported outcome measures (Thoma et al., 2012). 


\section{RESULTS}

All volunteers completed the study and neither systemic nor local side effects were noted during the entire study period. Biopsies in all volunteers were harvested, processed and could be used for analyses.

Descriptive histology and immunohistochemical analysis

The native, preoperatively obtained biopsies, revealed a continuous keratinized epithelium with long rete pegs, the subepithelial connective tissue was dense, regularly and horizontally arranged. Occasionally, a low number of lymphocytes and plasma cells were visible (Figure 4). The cytokeratin staining of the epithelium and the vimentin staining of the native subepithelial tissue were strong and even. In the factor VIII staining only few blood vessels were visible.

At day 4 , the original defect site was clearly visible and on the borders the preexisting epithelium remained (Figure $5 \mathrm{a}+\mathrm{b}$ ). The wound bed was characterized by a central concavity. The borders towards the surrounding native dense connective tissue were distinct. The defect area itself was predominantly occupied by moderate to large amounts of neutrophils and covered as well as interspersed by fibrin and acute bleedings. The cytokeratin staining was visible on the edges on the remaining epithelium and the vimentin staining and the Factor VIII staining were negative in these lesions. In XCM sites, the collagen matrix network was admixed with the fibrin.

At day 8 , the original defect was still visible, but on the edges the epithelium was beginning to grow over the defect as a thin layer with elongated epithelial cells. Keratinization was not fully established, even though some epithelial layers were present ( $\leq 2$ layers). The remaining part of the defect was filled by loose and sometimes strongly edematous young granulation tissue admixed with activated vessels, serous fluid and fibrin. A moderate to strong infiltration with neutrophils was observed. In case, the neutrophilic inflammation was strong, the granulation tissue was loose and less 
developed compared to the lesions with fewer neutrophils. The vessels were oriented $90^{\circ}$ to the defect site. In general, the control sites had more granulation tissue formation than XCM sites. Formation of epithelium was observed at the borders of the defect areas. In some sites, the transition between the native tissue and the newly formed tissue at the former biopsy site was not as smooth (Fig. 5c). In other sites, the transition was more continuous (Fig. 5d). Differences between test and control sites were difficult to detect. The epithelium at the borders and the preexisting epithelium were strongly positive in the cytokeratin staining. The vimentin staining was only faintly positive in the defect area demonstrating no new collagen formation up to this timepoint in both groups. The neutrophils were also positive in the vimentin staining. The activated and newly formed vessels expressed Factor VIII. The factor VIII staining showed more blood vessels close to the epithelium than in the underlying connective tissue and a larger number in XCM (Fig. 3a) than in control sites (Fig. 3b).

At day 15 , the defect area was covered by a thicker epithelium compared to the native biopsies taken at day 0 , showing irregular and misshapen rete pegs (longer and/or shorter and/or thicker) but already keratinization. The borders of the original wound were still visible, even though much less distinct. Great parts of the wound were replaced by a loose to dense connective tissue with a high number of blood vessels arranged $90^{\circ}$ to the former defect (granulation tissue). The loose connective tissue merged into the underlying native dense connective tissue. Intermingled in the granulation tissue a high amount of lymphocytes, few neutrophils and less plasma cells and eosinophils were visible. The XCM network could not be differentiated from the collagen fibers of the newly formed connective tissue at this time-point (Figure $5 \mathrm{e}+\mathrm{f}$ ). The XCM sites had a more pronounced inflammatory reaction than control sites. The cytokeratin staining of the epithelium was strong as was the staining of the connective tissue, but in a denser way than in the control sites.

At day 29 , the maturation had continued in both, the spongeous part and the superficial layer. Therefore, the borders of the original defect could hardly be identified. A shift had 
occurred and transformed the loose into a slightly dense granulation tissue with a lower number of newly formed blood vessels than at day 15 . However, the vessels were more prominent. The vessels were beginning to orient horizontally to the epithelium. Still, the density of the connective tissue did not catch up yet with the one from the preoperative time-point. At the epithelial layer, rete pegs had become more numerous and similar in dimension compared to the mature native tissue at least at the lateral aspects. The keratin layer reached dimensions almost similar to the original preoperative time-point. In the control sites, no inflammation was observed at all. In XCM sites, on the edges of the new collagen fibers, few multifocal lymphocytes and plasma cells were visible seldom arranged in follicles (Figure $5 \mathrm{~g}+\mathrm{h}$ ). The staining of the epithelium (cytokeratin) was strong, the staining of the connective tissue was stronger than that of the native tissue and the vessel density was less distinct than at day 15 as observed by the Factor VIII staining.

\section{Histomorphometric analyses}

Thickness of keratin layer and degree of epithelialization (Figure 1 in the appendix)

The thickness of the keratin layer continuously increased over 29 days. Whereas at day 4 , the majority of the wound surface was not covered by an epithelium, by day 15 , the entire surface was keratinized ( $>3$ layers). From day 15 to 29 , a further maturation of the keratin layer occurred. Similarly, the defect area covered with a keratinized epithelium continuously increased over 29 days. At day 4 , the percentage of the wound bed being keratinized was $12.4 \% \pm 7.5 \%$ (control) and $18.0 \% \pm 10.2 \%$ (XCM) and increased by roughly a $100 \%$ to day $8(19.7 \% \pm 25.5 \%$ control; $29.1 \% \pm 8.0 \% \mathrm{XCM})$. At day 15 , almost the entire wound bed was covered with a keratinized epithelium reaching values close to $100 \%$ without changes up to day 29 .

Quantitative analyses of regenerated tissues

Area 1 - superficial compartment (Figure 6 a (XCM); Figure 6 b (control)) 
At baseline, this region was predominantly ( $>50 \%$ occupied by a keratinized epithelium and, a dense and a loose connective tissue. From day 4 to day 8 , the amount of granulation tissue ( $32 \%$ to $88 \%$ control; $14 \%$ to $41 \% \mathrm{XCM}$ ) increased. In group XCM, the remaining collagen matrix made up for $25 \%$ and $51 \%$. At day 15 and 29 , in both groups, the epithelium already occupied more than $50 \%$ of area, followed by a loose connective tissue.

Area 2 - central compartment (Figure 2 in the appendix)

At baseline, a dense connective tissue was the predominant tissue (>70\%). At day 4 , similar to the superficial area, a granulation tissue was the predominant tissue, followed by the xenogeneic collagen matrix $(\mathrm{XCM})$. However, there was already a loose connective tissue that continuously increased in both groups up to day 15 ( $84 \%$ control; $62 \% \mathrm{XCM})$. The amount of dense connective tissue was higher at all time-points in group XCM.

Area 3 - apical compartment (Figure 3 in the appendix)

At baseline, the dense connective tissue accounted again for $>50 \%$ of the area, followed by fatty tissue (26\%) and a loose connective tissue (15\%). At day 4 and 8 , the main area was occupied by a loose connective tissue (13\%/9\% control; $25 \% / 43 \%$ XCM), a dense connective tissue $(21 \% / 22 \%$ control; $16 \% / 30 \% \mathrm{XCM})$ and the remaining xenogeneic collagen matrix $(10 \% / 8 \%)$. At day 29 , the tissues were similar to baseline with the exception of the missing fatty tissue. 


\section{DISCUSSION}

The present clinical study demonstrated a favorable tissue integration of a xenogeneic collagen matrix and an uneventful healing of control sites without any clinical complications during the entire study period. The early phase of wound healing was accelerated by the use of a XCM as a wound dressing. This was demonstrated by a reduced amount of granulation tissue and a higher degree of epithelialization. At 29 days, histologic analysis showed a regenerated palatal soft tissue similar to the native tissue for both treatment modalities.

Collagen membranes have been used for years for the purpose of guided tissue regeneration (Jung et al., 2013a). Integration and degradation processes of these membranes were analyzed using preclinical study designs with histologic outcome measures (von Arx et al., 2005). These findings can partially be applied on the more recently developed collagen matrices containing fibers of Collagen type I and III from the same origin but with a different organization.

Within the first 48 hours, there is a tissue reaction on the collagen material during the process of integration, explained by the presence of inflammatory cells (Rothamel et al., 2005). This tissue reaction depends on the collagen organization within the devices. The closer the collagen organization in the devices mimics natural collagen fiber organization, the less pronounced are inflammatory reactions. Nevertheless, within the same healing period, an early attachment of epithelial cells and fibroblasts spreading all over the xenogeneic collagen matrix was detected, proving biocompatibility of the matrix (Willershausen et al., 2014, Ghanaati et al., 2011). During this first phase, the matrix was still cell occlusive, similar to a membrane. This was demonstrated by cells primarily growing along the surface of the dense matrix layer. The ingrowth pattern of endothelial cells and blood vessels into collagen membranes was investigated previously (Schwarz et al., 2008, Schwarz et al., 2006). In these studies, collagen membranes were implanted without contact to the oral environment. Angiogenesis all through the 
collagen membrane was shown earliest at 1 week. In the present study with an open healing design, collagen matrix degradation patterns were different. Interestingly, angiogenesis was observed at a similar time-point ( 8 days) as demonstrated by endothelial cells marked by rabbit anti Factor VIII. This might be explained by the fact that these matrices consist of a cell-occlusive superficial layer and a more spongeous layer underneath.

Preclinical data are available for different collagen devices in terms of degradation processes with submerged healing protocols or wound healing in contact with the oral environment (Vignoletti et al., 2015, Rocchietta et al., 2012, von Arx et al., 2005). In an experimental study in cats, collagen matrices were either placed into a pocket for submerged healing or were exposed to the oral environment (Tal et al., 2008). Both modalities healed uneventfully, but the degradation process was faster in open healing sites. These findings are in line with the present study, demonstrating no remnants of the xenogeneic collagen matrix at 15 days and a fast regeneration of the connective tissue and the epithelium. Inter-individual differences, however, were observed with some volunteers presenting a faster healing than others. This might be attributed to the fact that the protective splint provided to the volunteers and instructed to be used during the first day and in case of pain, was not used on a regular and standardized basis. Some volunteers might have used the stent for a day, whereas others made use of the splint for an even longer period of time. The influence of wearing a protective splint on the early healing remains uncertain as this was not evaluated, but would have affected both, control and test sites.

In terms of keratinization of the wound, XCM sites reached roughly $30 \%$ more keratinized tissue at day 4 and 8 . However, the differences between the groups at these time-points were small. At day 15 , the surface in all sites was keratinized. Due to the selection of the time-points of biopsy harvesting and the limited number of biopsies obtained, the present results cannot fully formulate a trend in terms of the keratinization process. Further investigations using the same experimental design are 
needed to evaluate the extent of keratinization at 10-12 days after surgery. A positive effect of a prototype XCM on keratinization has previously been found in vitro (Nocini et al., 2013). The effect was explained on a molecular basis with the expression of Interleukin-6 (Il-6) by keratinocytes when attaching to the collagen fibrils of the matrix. Il-6 acts as an anti-apoptosis molecule, protecting the cells of their spontaneous apoptosis, thereby accelerating the colonization.

The quantitative analysis in the present study revealed no differences between the groups in the apical compartment, consisting mainly of connective and fatty tissue. The amount of connective and fatty tissue in this compartment remained unchanged during the entire study period in both groups. Probably, this compartment was only partially removed when the first biopsy was harvested. The XCM mainly influenced the wound healing superficially by facilitating epithelial cell migration. The greatest differences between XCM and spontaneous healing sites were found in the superficial and central compartment. Most remarkably, at day 4 as well as at day 8 , more granulation tissue was formed in sites without a collagen matrix. The percentage of residual collagen matrix corresponded to the reduced amount of granulation tissue in both compartments at both time-points. Since the cellular processes of early angiogenesis and cell migration within the matrix are not fully understood yet, this finding might be an explanation for the accelerated wound healing within the first days. In general, wound healing can be divided into three stages, inflammation, tissue formation and tissue remodeling (Singer and Clark, 1999). In the early phase of an open wound, the body's primary objective is to obtain a seal against outside to prevent the invasion of microorganisms. As a normal act of quickest possible wound closure, granulation tissue formation occurs. Subsequently, the phases of tissue formation and tissue remodeling finally lead to a regeneration and/or reparation of the lesion. This means, that the granulation tissue subsequently needs to be reorganized and rebuilt during the second and the third phase. Assuming that the xenogeneic collagen matrix acts as a matrix (spongeous 
layer) for subsequent cell ingrowth and connective tissue formation, it could, at an early stage, reduce granulation tissue formation, and secondly facilitate tissue formation.

The translation of the obtained histological findings into the clinics suggests that the application of a collagen matrix as a seal for open palatal wound might be beneficial. This is primarily related to the early stages of wound healing ( 4 and 8 days). Similar findings were obtained in an earlier study (Thoma et al., 2012). Furthermore, clinical studies demonstrated a good color adaption (Schmitt et al., 2015, Sanz et al., 2009). However, data in terms of patient-reported outcome measures assessing general morbidity remain scarce in the literature. Less postoperative sensitivity was detected at 4 and 8 days when the xenogeneic collagen matrix was applied. Morbidity, however, was assessed locally (Thoma et al., 2012). 


\section{CONCLUSIONS}

The application of a xenogeneic collagen matrix (XCM) as a wound dressing on palatal wounds might be beneficial during the early stages of wound healing. This finding was predominantly observed at 4 and 8 days after surgery and might be due to the space maintenance of the XCM and less granulation tissue formation compared to spontaneously healing sites. However, further research with a larger sample size is needed to confirm these results obtained by an experimental design. 


\section{ACKNOWLEDGEMENTS AND CONFLICT OF INTEREST}

The authors gratefully acknowledge the help and support of Sonja Hitz (Clinic of Fixed and Removable Prosthodontics and Dental Material Science, University of Zurich, Switzerland) for the preparation and analyses of the histologic slides. The study was supported by the Clinic of Fixed and Removable Prosthodontics and Dental Material Science, University of Zurich, Switzerland and by a research grant of Geistlich Pharma AG, Wolhusen, Switzerland. The authors report no conflicts of interests. 


\section{Figure Legend}

Figure $\mathbf{1} \mathbf{a}+\mathbf{b}$ Punch biopsy harvesting by means of a digitally designed stent (left) and illustrative picture taken after harvesting the first biopsy with a diameter of $6 \mathrm{~mm}$ and application of the collagen matrix (right). The stent for the second biopsy with a diameter of $8 \mathrm{~mm}$ will allow the removal of the wound margins of the primary lesion.

Figure 2 Region of interest (ROI) (green rectangle) positioned along the surface of the harvested biopsy (collagen matrix $=\mathrm{XCM}$ ), containing a superficial, central and apical compartment. $\mathrm{DCT}=$ dense connective tissue; $\mathrm{LCT}=$ loose connective tissue; $\mathrm{E}=$ epithelium; FT = fatty tissue; GT = granulation tissue; $\mathrm{KL}=$ keratinized layer .

Figure 3 Immunohistochemical staining with endothelial cells marked by Factor VIII at day 8 . Test site $(X C M=$ collagen matrix; a) and control site $(b) . B V=$ blood vessel; $E=$ Epithelium; MSL= matrix, spongeous layer.

Figure 4 Biopsy containing native tissue of a test site ( $\mathrm{XCM}=$ collagen matrix). A matured epithelium (E) and keratinized layer $(K L)$, high amounts of dense connective tissue (DCT) and fatty tissue (FT) and low amounts of loose connective tissue (LCT) are visible.

Figure $\mathbf{5}$ a-h At 4 days, the collagen matrix (a) is already well integrated, whereas spontaneous healing (b) shows high amounts of granulation tissue (GT) superficially. A distinct wound margin towards the keratinized epithelium (E) is visible. The spongeous layer of the matrix (MSL) shows an ingrowth of GT, the dense layer (MDL) is clearly stained. At 8 days, there are still remnants of the matrix visible (c) compared to a higher amount of GT at spontaneously healing sites (d). Wound margins are less distinct and an ingrowth of $E$ is visible. Loose connective tissue (LCT) formation takes place in the 
central compartment. At 15 days, a continuous keratinized layer $(\mathrm{KL})$ covers the newly structured loose connective tissue (LCT) in the sites with a collagen matrix (e) as well as in the spontaneously healed sites (f). At 29 days, both, the site treated with a collagen matrix $(\mathrm{g})$ and the spontaneously healed site $(\mathrm{h})$ show a further maturation of $\mathrm{E}$ and $\mathrm{KL}$. Apart of the lower amount of dense connective tissue (DCT), compensated by a higher amount of loose connective tissue (LCT), there are only minor differences compared to the native tissue in Figure 4.

Figure $\mathbf{6} \mathbf{a}+\mathbf{b}$ Histomorphometric results of the collagen matrix group (left) and of the spontaneous healing group (right) in the superficial compartment at the 5 different timepoints.

Figure 1 Appendix Boxplots illustrating the amount of keratinization in \% evaluated at the four different time-points after the first biopsy. The surface was considered to be keratinized if at least 3 layers were present. $\mathrm{XCM}=$ xenogeneic collagen matrix; $\mathrm{C}=$ control, spontaneous healing.

Figure $\mathbf{2} \mathbf{a}+\mathbf{b}$ Appendix Histomorphometric results of the collagen matrix group (left) and of the spontaneous healing group (right) in the central compartment at the 5 different time-points.

Figure $\mathbf{3} \mathbf{a}+\mathbf{b}$ Appendix Histomorphometric results of the collagen matrix (left) and the spontaneous healing group (right) in the apical compartment at the 5 different timepoints. 


\section{REFERENCES}

Cairo, F., Nieri, M. \& Pagliaro, U. (2014) Efficacy of periodontal plastic surgery procedures in the treatment of localized facial gingival recessions. A systematic review. Journal of Clinical Periodontology 41 Suppl 15, S44-62. doi:10.1111/jcpe.12182.

Cardaropoli, D., Tamagnone, L., Roffredo, A. \& Gaveglio, L. (2012) Treatment of gingival recession defects using coronally advanced flap with a porcine collagen matrix compared to coronally advanced flap with connective tissue graft: a randomized controlled clinical trial. Journal of Periodontology 83, 321-328. doi:10.1902/jop.2011.110215.

Ghanaati, S., Schlee, M., Webber, M. J., Willershausen, I., Barbeck, M., Balic, E., Gorlach, C., Stupp, S. I., Sader, R. A. \& Kirkpatrick, C. J. (2011) Evaluation of the tissue reaction to a new bilayered collagen matrix in vivo and its translation to the clinic. Biomed Mater 6, 015010. doi:10.1088/1748-6041/6/1/015010.

Griffin, T. J., Cheung, W. S., Zavras, A. I. \& Damoulis, P. D. (2006) Postoperative complications following gingival augmentation procedures. Journal of Periodontology 77, 2070-2079.

Harris, R. J., Miller, R., Miller, L. H. \& Harris, C. (2005) Complications with surgical procedures utilizing connective tissue grafts: a follow-up of 500 consecutively treated cases. International Journal of Periodontics and Restorative Dentistry 25, 449-459.

Jung, R. E., Fenner, N., Hammerle, C. H. \& Zitzmann, N. U. (2013a) Long-term outcome of implants placed with guided bone regeneration (GBR) using resorbable and non-resorbable membranes after 12-14 years. Clinical Oral Implants Research 24, 1065-1073. doi:10.1111/j.1600-0501.2012.02522.x.

Jung, R. E., Hurzeler, M. B., Thoma, D. S., Khraisat, A. \& Hammerle, C. H. (2011) Local tolerance and efficiency of two prototype collagen matrices to increase the width 
of keratinized tissue. Journal of Clinical Periodontology 38, 173-179. doi:10.1111/j.1600-051X.2010.01640.x.

Jung, R. E., Philipp, A., Annen, B. M., Signorelli, L., Thoma, D. S., Hammerle, C. H., Attin, T. \& Schmidlin, P. (2013b) Radiographic evaluation of different techniques for ridge preservation after tooth extraction: a randomized controlled clinical trial. Journal of Clinical Periodontology 40, 90-98. doi:10.1111/jcpe.12027.

Kim, D. M. \& Neiva, R. (2015) Periodontal soft tissue non-root coverage procedures: a systematic review from the AAP regeneration workshop. Journal of Periodontology 86, S56-72. doi:10.1902/jop.2015.130684.

Levine, R. A., Huynh-Ba, G. \& Cochran, D. L. (2014) Soft tissue augmentation procedures for mucogingival defects in esthetic sites. International Journal of Oral and Maxillofacial Implants 29 Suppl, 155-185. doi:10.11607/jomi.2014suppl.g3.2.

Liu, J., Bian, Z., Kuijpers-Jagtman, A. M. \& Von den Hoff, J. W. (2010) Skin and oral mucosa equivalents: construction and performance. Orthod Craniofac Res 13, 1120. doi:10.1111/j.1601-6343.2009.01475.x.

Lorenzo, R., Garcia, V., Orsini, M., Martin, C. \& Sanz, M. (2012) Clinical efficacy of a xenogeneic collagen matrix in augmenting keratinized mucosa around implants: a randomized controlled prospective clinical trial. Clinical Oral Implants Research 23, 316-324. doi:10.1111/j.1600-0501.2011.02260.x.

Nevins, M., Nevins, M. L., Kim, S. W., Schupbach, P. \& Kim, D. M. (2011) The use of mucograft collagen matrix to augment the zone of keratinized tissue around teeth: a pilot study. International Journal of Periodontics and Restorative Dentistry 31, 367-373.

Nocini, P. F., Zanotti, G., Castellani, R., Grasso, S., Cristofaro, M. G. \& De Santis, D. (2013) Bi-layered collagen nano-structured membrane prototype (collagen matrix 10826((R))) for oral soft tissue regeneration: an "in vitro" study. Clinical Oral Implants Research 24, 612-617. doi:10.1111/j.1600-0501.2012.02427.x. 
Rocchietta, I., Schupbach, P., Ghezzi, C., Maschera, E. \& Simion, M. (2012) Soft tissue integration of a porcine collagen membrane: an experimental study in pigs. International Journal of Periodontics and Restorative Dentistry 32, e34-40.

Rothamel, D., Schwarz, F., Sager, M., Herten, M., Sculean, A. \& Becker, J. (2005) Biodegradation of differently cross-linked collagen membranes: an experimental study in the rat. Clinical Oral Implants Research 16, 369-378. doi: $10.1111 / j .1600-0501.2005 .01108 . x$.

Rotundo, R. \& Pini-Prato, G. (2012) Use of a new collagen matrix (mucograft) for the treatment of multiple gingival recessions: case reports. International Journal of Periodontics and Restorative Dentistry 32, 413-419.

Sanz, M., Lorenzo, R., Aranda, J. J., Martin, C. \& Orsini, M. (2009) Clinical evaluation of a new collagen matrix (Mucograft prototype) to enhance the width of keratinized tissue in patients with fixed prosthetic restorations: a randomized prospective clinical trial. Journal of Clinical Periodontology 36, 868-876. doi:10.1111/j.1600051X.2009.01460.x.

Schmitt, C. M., Moest, T., Lutz, R., Wehrhan, F., Neukam, F. W. \& Schlegel, K. A. (2015) Long-term outcomes after vestibuloplasty with a porcine collagen matrix (Mucograft ) versus the free gingival graft: a comparative prospective clinical trial. Clinical Oral Implants Research. doi:10.1111/clr.12575.

Schmitt, C. M., Tudor, C., Kiener, K., Wehrhan, F., Schmitt, J., Eitner, S., Agaimy, A. \& Schlegel, K. A. (2013) Vestibuloplasty: porcine collagen matrix versus free gingival graft: a clinical and histologic study. Journal of Periodontology 84, 914923. doi:10.1902/jop.2012.120084.

Schwarz, F., Rothamel, D., Herten, M., Sager, M. \& Becker, J. (2006) Angiogenesis pattern of native and cross-linked collagen membranes: an immunohistochemical study in the rat. Clinical Oral Implants Research 17, 403-409. doi:10.1111/j.1600-0501.2005.01225.x.

Schwarz, F., Rothamel, D., Herten, M., Wustefeld, M., Sager, M., Ferrari, D. \& Becker, J. (2008) Immunohistochemical characterization of guided bone regeneration at a 
dehiscence-type defect using different barrier membranes: an experimental study in dogs. Clinical Oral Implants Research 19, 402-415. doi:10.1111/j.16000501.2007.01486.x.

Simion, M., Rocchietta, I., Fontana, F. \& Dellavia, C. (2012) Evaluation of a resorbable collagen matrix infused with rhPDGF-BB in peri-implant soft tissue augmentation: a preliminary report with 3.5 years of observation. International Journal of Periodontics and Restorative Dentistry 32, 273-282.

Singer, A. J. \& Clark, R. A. (1999) Cutaneous wound healing. New England Journal of Medicine 341, 738-746. doi:10.1056/NEJM199909023411006.

Tal, H., Kozlovsky, A., Artzi, Z., Nemcovsky, C. E. \& Moses, O. (2008) Cross-linked and non-cross-linked collagen barrier membranes disintegrate following surgical exposure to the oral environment: a histological study in the cat. Clinical Oral Implants Research 19, 760-766.

Thoma, D. S., Benic, G. I., Zwahlen, M., Hammerle, C. H. \& Jung, R. E. (2009) A systematic review assessing soft tissue augmentation techniques. Clinical Oral Implants Research 20 Suppl 4, 146-165. doi:10.1111/j.16000501.2009.01784.x.

Thoma, D. S., Buranawat, B., Hammerle, C. H., Held, U. \& Jung, R. E. (2014) Efficacy of soft tissue augmentation around dental implants and in partially edentulous areas: a systematic review. Journal of Clinical Periodontology 41 Suppl 15, S7791. doi:10.1111/jcpe.12220.

Thoma, D. S., Jung, R. E., Schneider, D., Cochran, D. L., Ender, A., Jones, A. A., Gorlach, C., Uebersax, L., Graf-Hausner, U. \& Hammerle, C. H. (2010) Soft tissue volume augmentation by the use of collagen-based matrices: a volumetric analysis. Journal of Clinical Periodontology 37, 659-666. doi:10.1111/j.1600051X.2010.01581.x.

Thoma, D. S., Sancho-Puchades, M., Ettlin, D. A., Hammerle, C. H. \& Jung, R. E. (2012) Impact of a collagen matrix on early healing, aesthetics and patient morbidity in 
oral mucosal wounds - a randomized study in humans. Journal of Clinical Periodontology 39, 157-165. doi:10.1111/j.1600-051X.2011.01823.x.

Vignoletti, F., Nunez, J., de Sanctis, F., Lopez, M., Caffesse, R. \& Sanz, M. (2015) Healing of a xenogeneic collagen matrix for keratinized tissue augmentation. Clinical Oral Implants Research 26, 545-552. doi:10.1111/clr.12441.

von Arx, T., Broggini, N., Jensen, S. S., Bornstein, M. M., Schenk, R. K. \& Buser, D. (2005) Membrane durability and tissue response of different bioresorbable barrier membranes: a histologic study in the rabbit calvarium. International Journal of Oral and Maxillofacial Implants 20, 843-853.

Wessel, J. R. \& Tatakis, D. N. (2008) Patient outcomes following subepithelial connective tissue graft and free gingival graft procedures. Journal of Periodontology 79, 425430.

Willershausen, I., Barbeck, M., Boehm, N., Sader, R., Willershausen, B., Kirkpatrick, C. J. \& Ghanaati, S. (2014) Non-cross-linked collagen type I/III materials enhance cell proliferation: in vitro and in vivo evidence. J Appl Oral Sci 22, 29-37. doi: $10.1590 / 1678-775720130316$. 


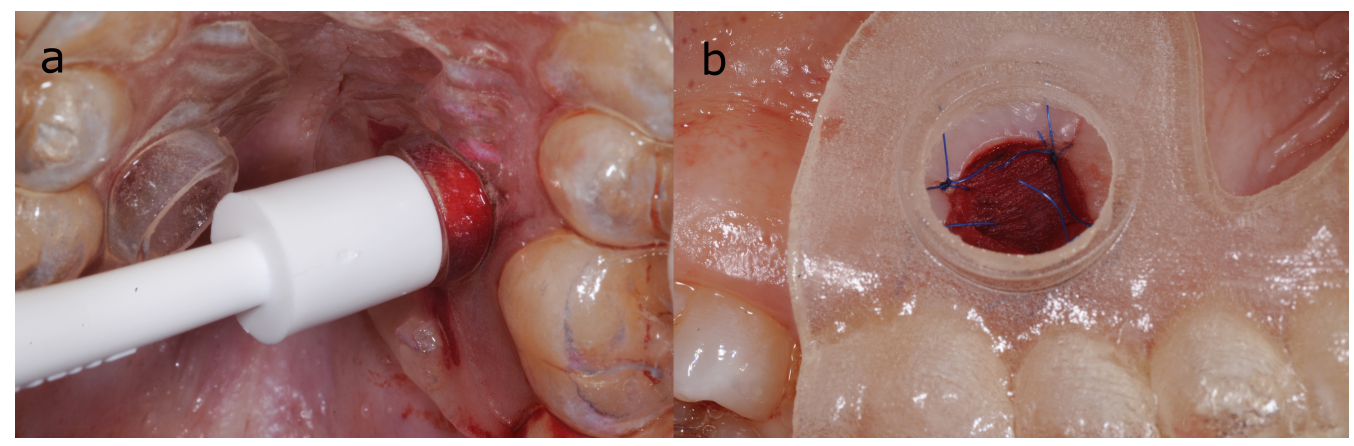

Figure 1

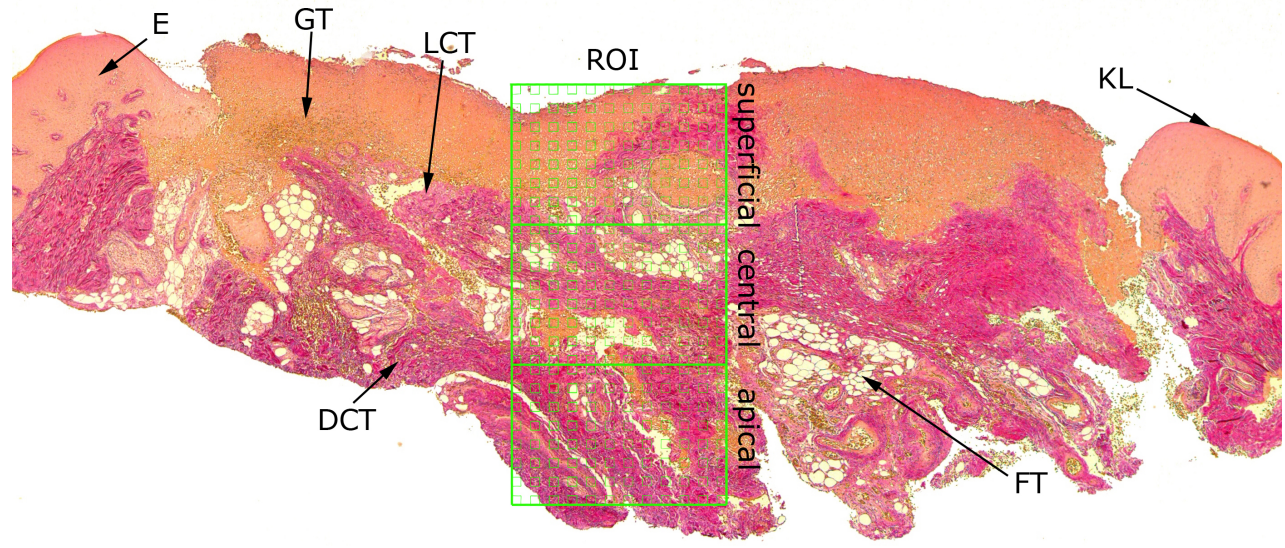

Figure 2

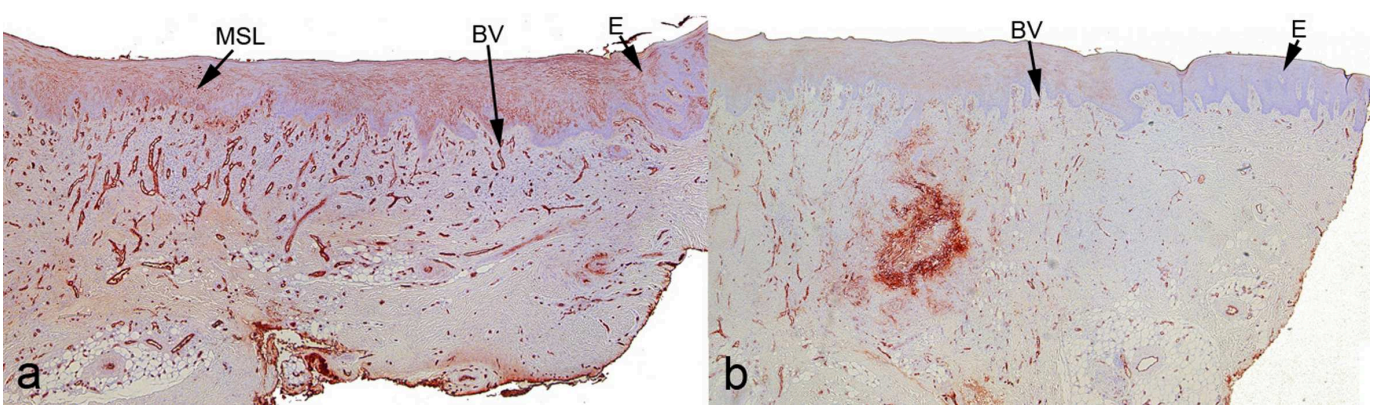

Figure 3 


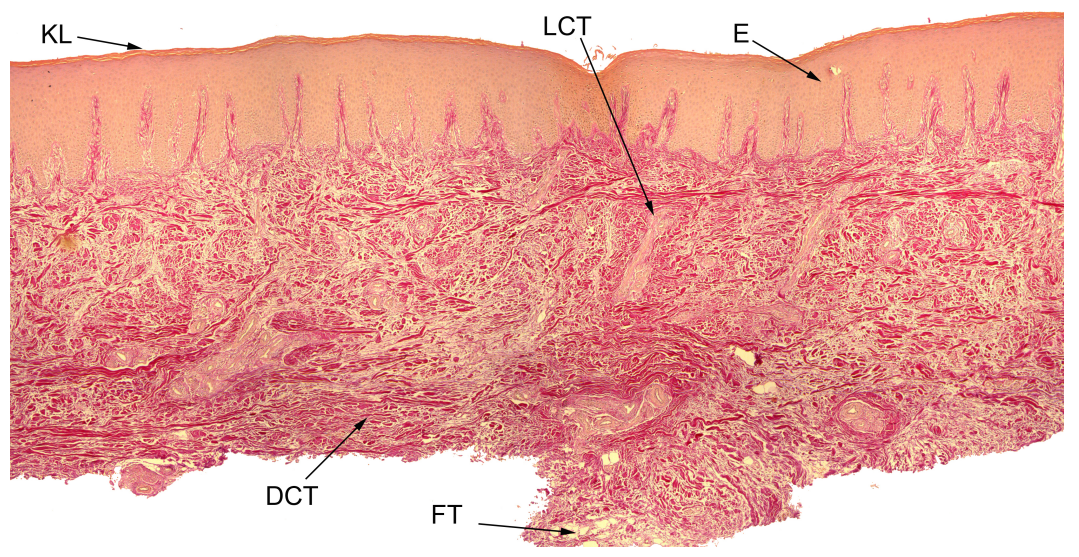

Figure 4
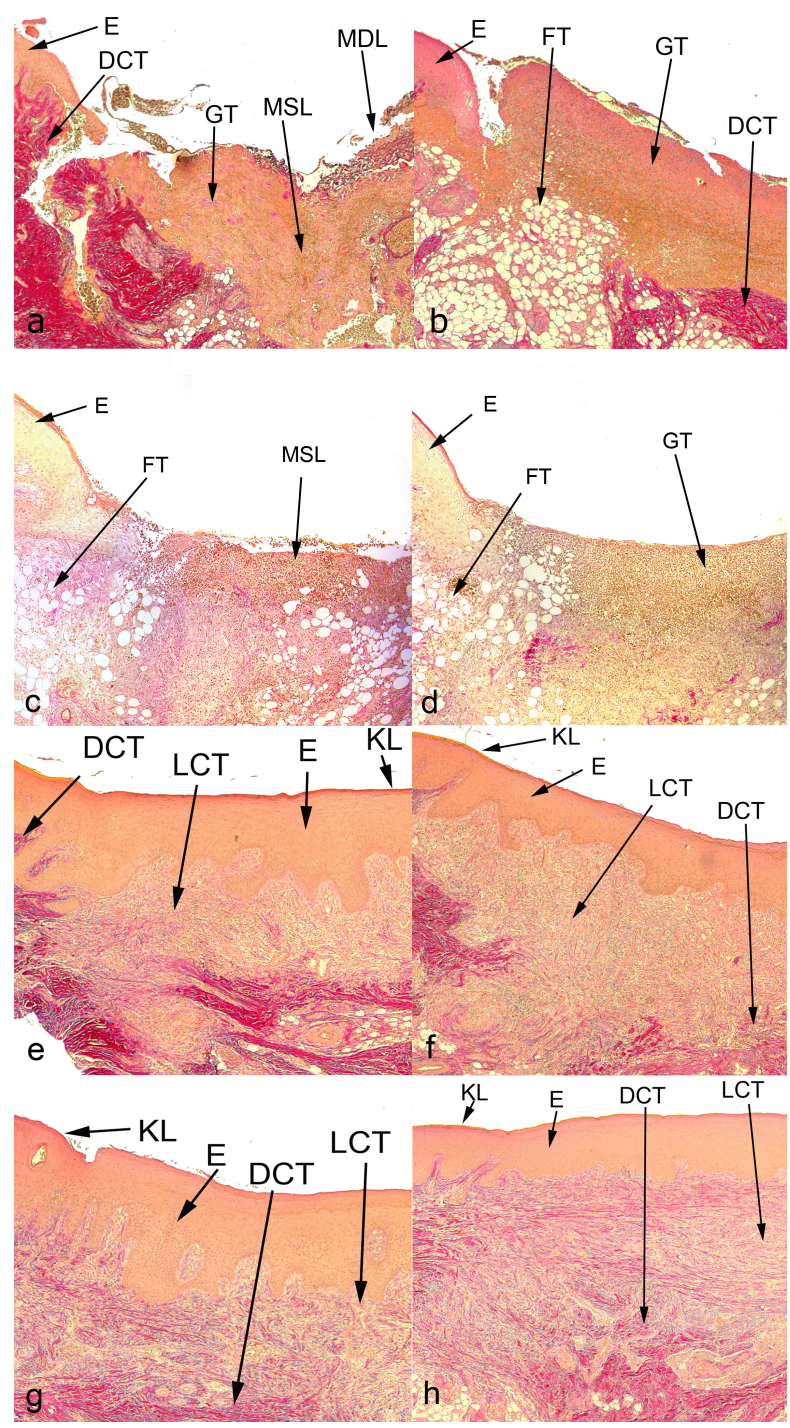

Figure 5 
a (XCM)

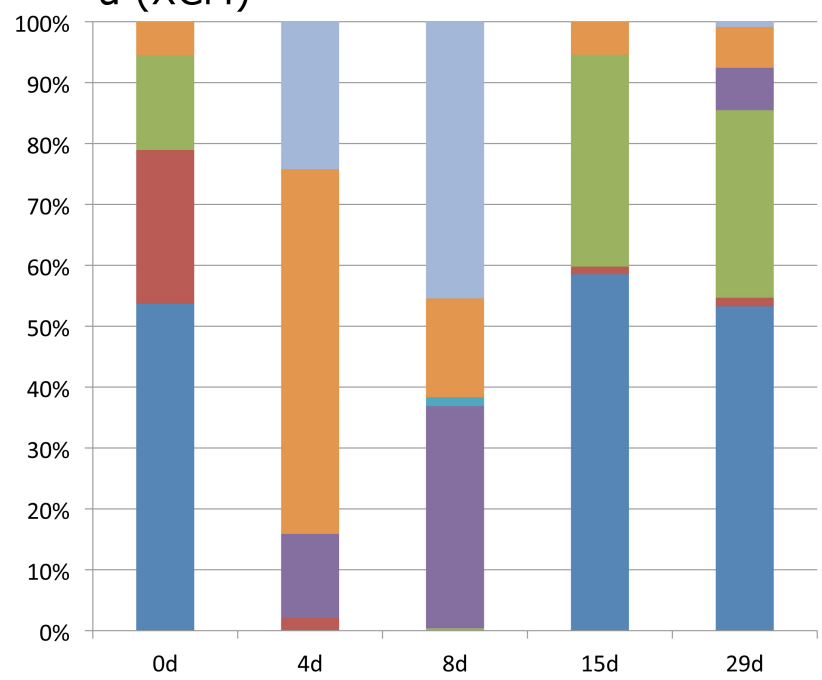

b (control)

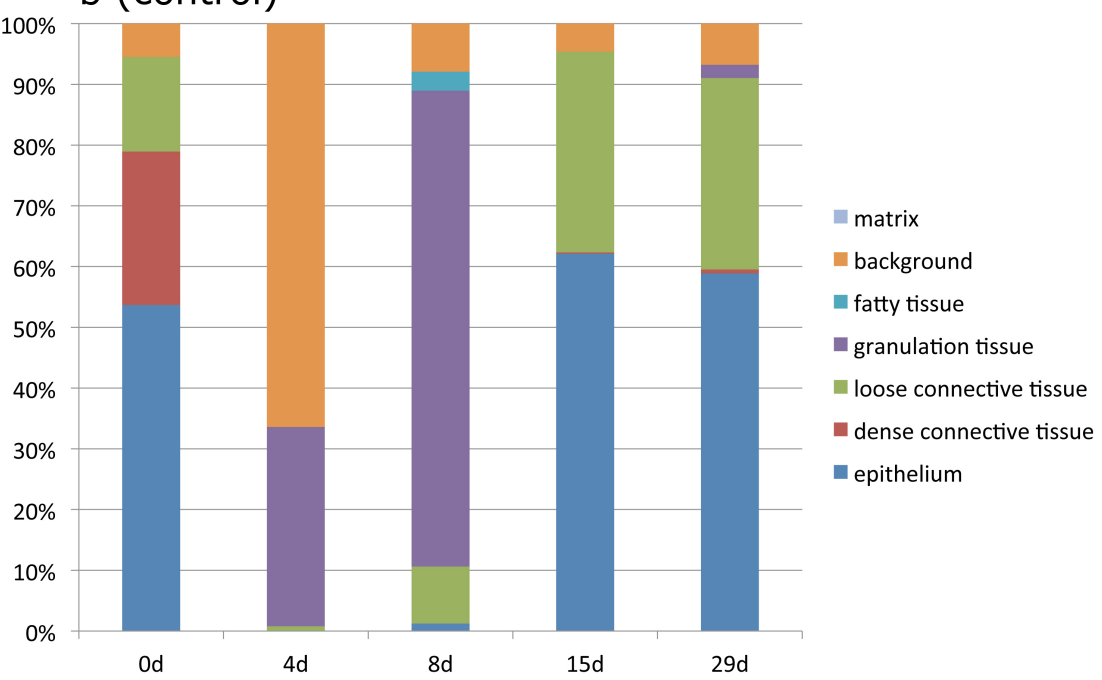

Figure 6 


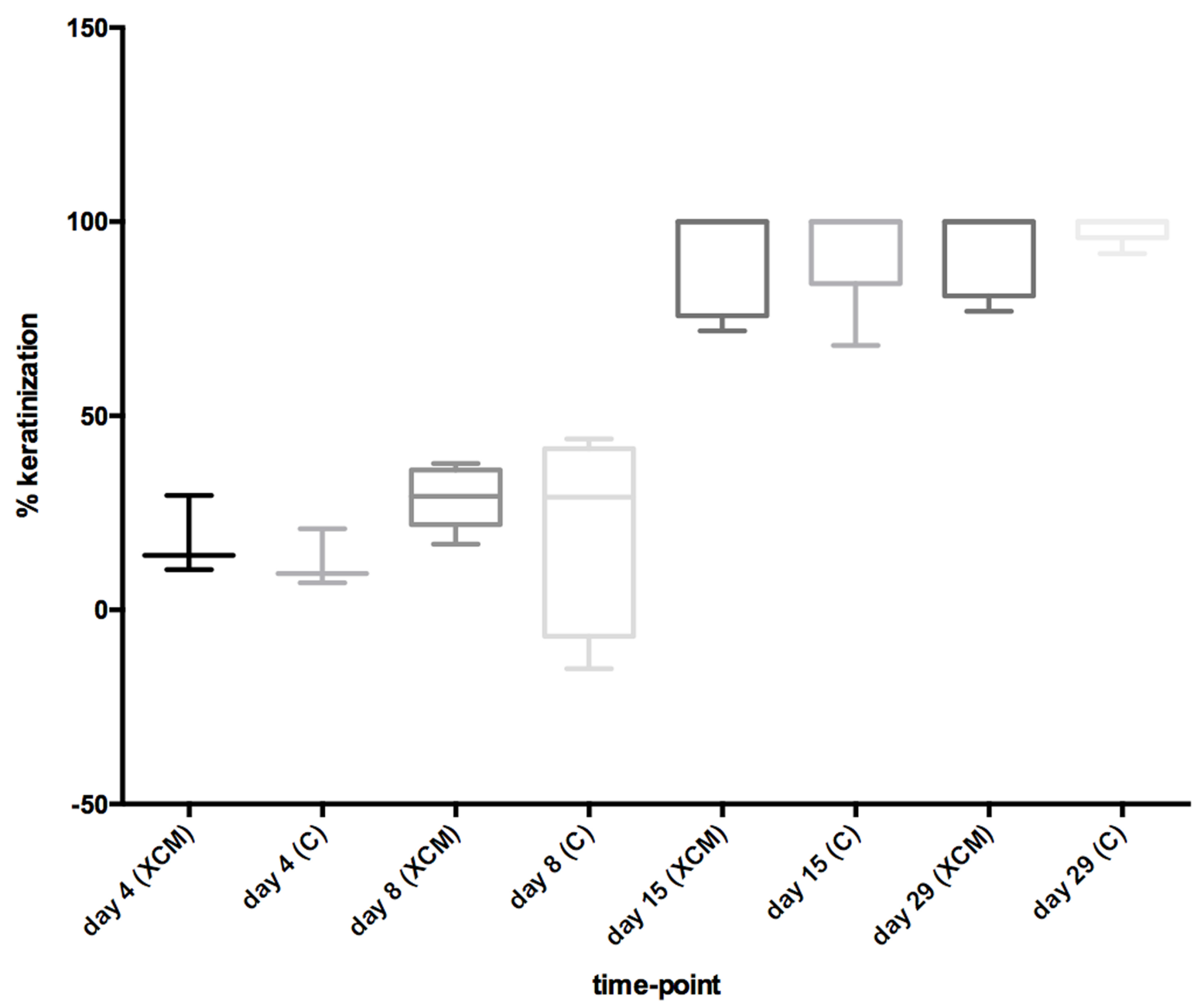

Appendix 1
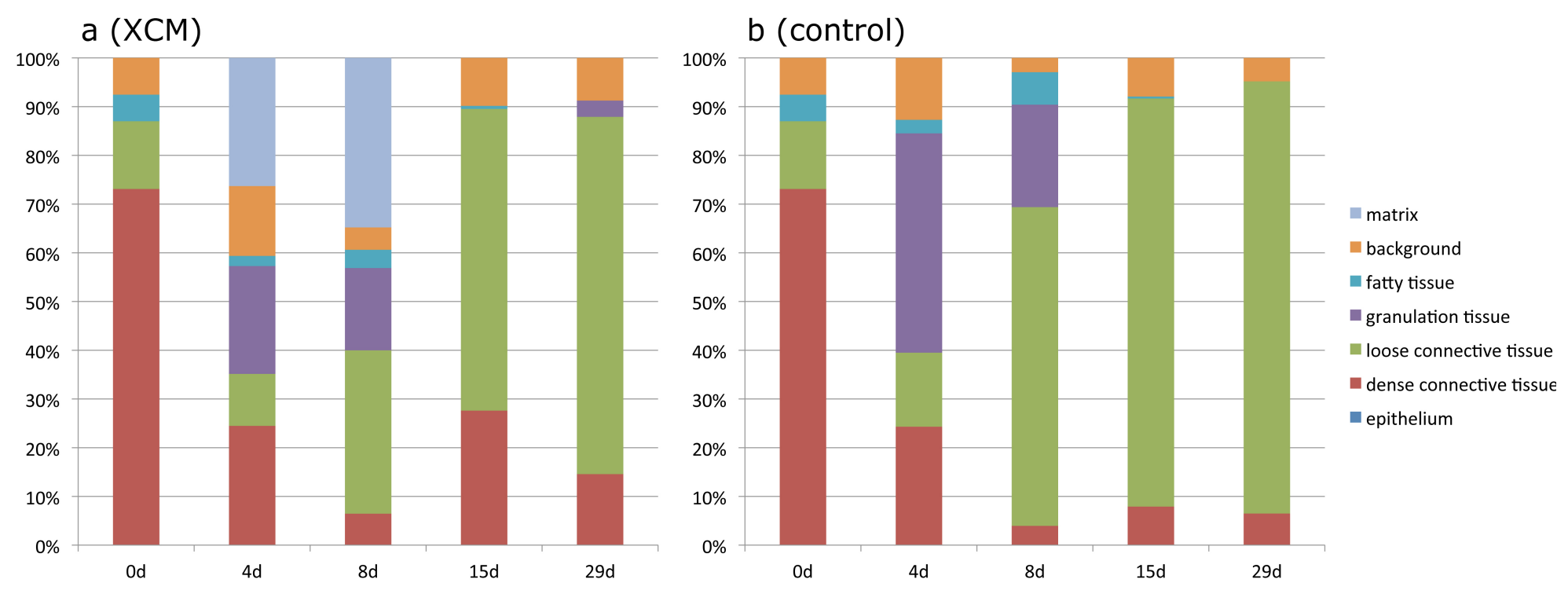

Appendix 2 
a (XCM)

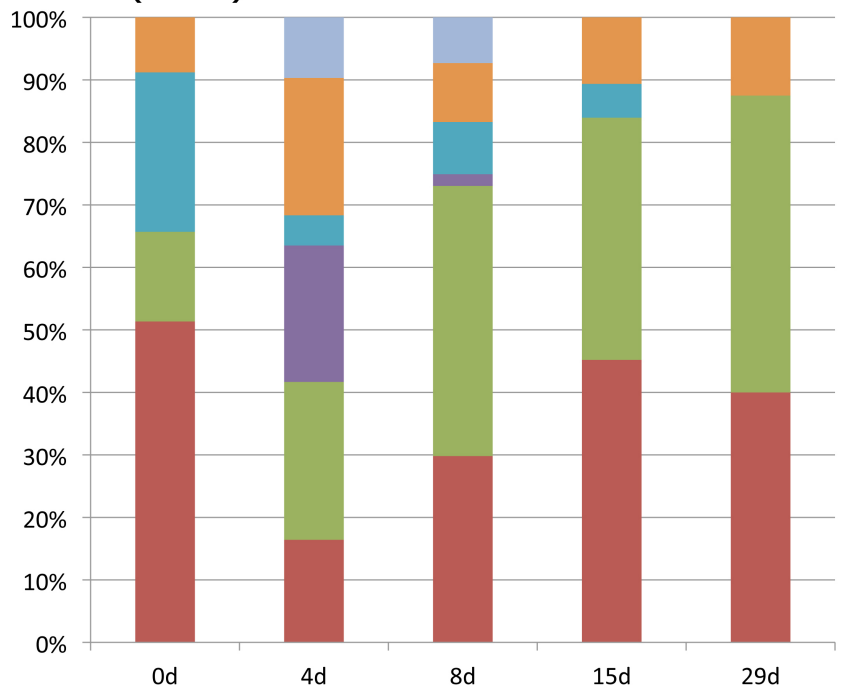

b (control)

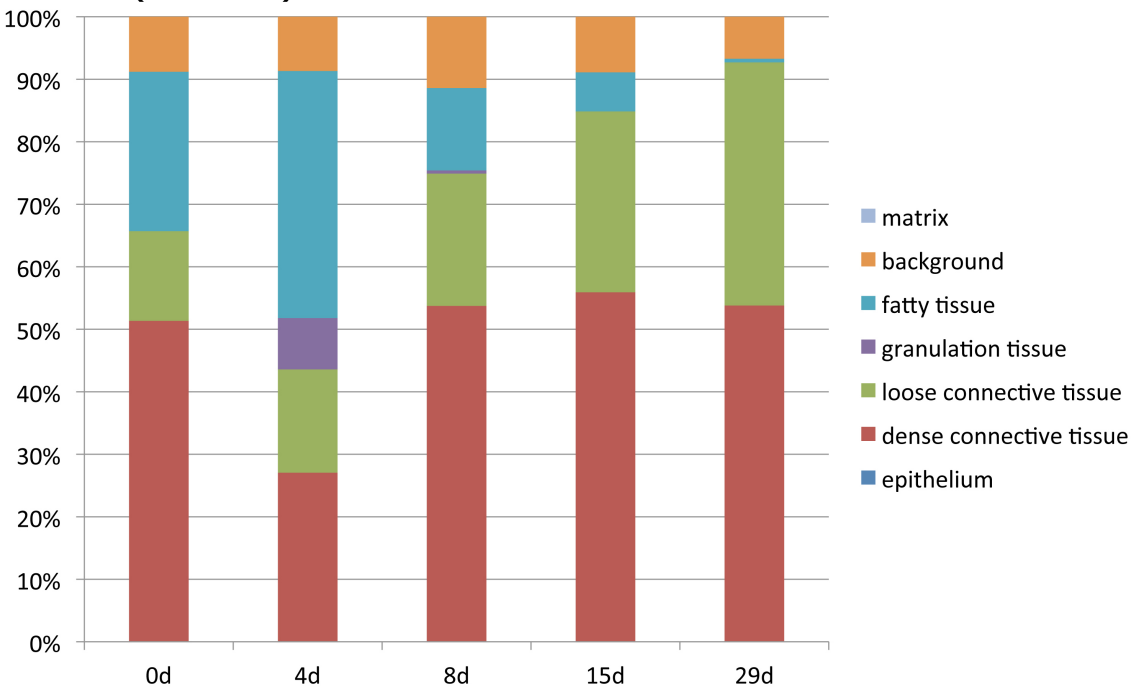

Appendix 3 